\title{
Surgical Stabilization of Pelvic and Acetabular Fractures: A Review on the Determinants of Clinical Outcomes
}

\author{
SS Sathappan, FRCS Ortho, CM Qi, MBBS, A Pillai, MRCS (Edin) \\ Department of Orthopedic Surgery, Tan Tock Seng Hospital, Singapore
}

\begin{abstract}
Aim: Pelvic and acetabular fractures are associated with high-energy trauma. The aim of this study was to identify factors that are associated with specific clinical outcomes following treatment for these fractures. Methods: A consecutive series of 30 patients who had surgical intervention for either pelvic or acetabular fractures formed the sample for this study. Clinical variables reviewed were: age, associated injuries, number of surgical procedures, time to surgery and post-operative complications. Clinical outcomes were assessed using Matta's grading of postoperative fracture reduction alignment, and functional outcomes were graded using D'Aubigne \& Postel's Hip scoring system. Results: Study subjects included twelve pelvic fractures and eighteen acetabular fractures. Patients older than 50 years of age had poorer hip scores despite surgery. Earlier fracture fixation (within five days) was associated with better hip scores. Patients with acetabular fractures generally had better functional outcomes than patients with pelvic fractures (mean hip score 15.0 vs. 13.5). Closer anatomical reduction of acetabular fractures was associated with better functional outcome. Conclusion: Improved clinical outcomes are associated with younger age, fewer concomitant injuries, shorter time interval to surgery and more closely approximated anatomical fracture reduction.
\end{abstract}

Key Words:

Pelvic Fracture, Acetabular Fracture, Surgical Stabilization, Anatomical Reduction, Clinical Outcomes

\section{INTRODUCTION}

Surgical management of pelvic and acetabular fractures is one of the most challenging facets of orthopaedic trauma. Such injuries typically result from either a fall from height or from motor vehicle accidents ${ }^{1}$. Due to the high-energy transfer involved during impact, a significant number of these patients present with polytrauma and often pose diagnostic and surgical dilemmas to the managing orthopaedic surgeon.

Pelvic fracture patients often present with unstable physiology and are considered a surgical emergency ${ }^{2}$.
Acetabular fractures should be anatomically reduced since there is an inherent risk of eventual osteoarthrosis ${ }^{3}$. In fact, early anatomical reduction of both these types of fractures is essential for optimal functional recovery in these patients ${ }^{4,5,6}$.

This clinical study was undertaken to review a consecutive case series of patients undergoing surgical fixation of pelvic and acetabular fractures, the aim being a thorough clinical review of the epidemiological data of such patients and clinical determinants of surgical outcome.

\section{MATERIALS AND METHODS}

A retrospective review was conducted in a consecutive series of 30 patients undergoing surgical fixation of pelvic and acetabular fractures at the authors' institution over a 4-year time period. Patients who were treated with conservative nonsurgical management of pelvic or acetabular fractures were excluded from this study.

Patients with isolated orthopaedic injuries were directly admitted under the care of an orthopaedic surgeon. However, patients with multiple non-orthopaedic injuries (e.g. visceral trauma, head injury, etc.) were admitted under the care of general surgeons and comanaged by orthopaedic surgeons. Fracture characterization was conducted using standard preoperative radiographs (anteroposterior pelvis, inlet \& outlet views of the pelvis, and Judet's view) and computed tomography (CT) with 3-D reconstruction. Clinical preoperative stabilisation of patients was ensured by anaesthetists.

All surgical procedures were performed by senior orthopaedic surgeons. Following anaesthesia induction, broad-spectrum antibiotics were administered prophylactically and continued postoperatively until all surgical drains were removed. Postoperatively, patients were monitored either in a critical care unit (CCU) or an orthopaedic ward based on physiological parameters as assessed by the anaesthetists. All patients were given antithrombotic stockings and were administered low molecular weight heparin for prophylaxis against deep vein thrombosis (DVT). When patients attained satisfactory ambulatory status they were discharged home or to an outpatient rehabilitation facility.

Corresponding Author: SS Sathappan, Consultant \& Clinician Scientist, Adult Reconstructive Surgery \& Complex Trauma, Department of Orthopedic Surgery, Tan Tock Seng Hospital, 11 Jalan Tan Tock Seng, Singapore 308433 Email: Sathappan@ttsh.com.sg 
Following discharge, patients were reviewed at 6 weeks, 3 months, 6 months, 12 months and then yearly. At each outpatient review, standard radiographs of the pelvis were repeated. CT scans were repeated only in the event of complications. Acetabular fracture fixation as well as pelvic fixation techniques were critically reviewed using a modified version of the criteria described by Matta et al. ${ }^{7,8}$. This involved the summation of the fracture displacement on 2 plain radiographic views. These values were obtained for both preoperative and postoperative radiographs (Table 1a). The author who was not involved in the original surgical procedure performed all data retrieval and analysis (for both preoperative and postoperative data). Preoperative factors studied included the patient's age and gender, mechanism of injury, fracture classification, associated injuries and time to surgical intervention ${ }^{9,10}$. Postoperative factors reviewed included: duration of stay in $\mathrm{CCU}$, complications and ambulatory status at the most recent follow-up.

Functional outcome of patients with pelvic and acetabular fractures was graded using the D'Aubigne \& Postel's Hip scoring system (Table 1b). Functional outcome scores were correlated to the various preoperative and postoperative factors using the Chi-squared test at 5\% level of significance.

\section{RESULTS}

There were a total of 30 patients who underwent surgical fixation for pelvic or acetabular fractures over the four year time period studied here. There were 9 females and 21 males. The mean and median age at time of surgery was $38 \mathrm{y}$ (range, $22-69 y)$ and $30 y$ for females and males respectively. The majority of the patients were young and $87 \%$ (26 patients) had no pre-existing co-morbidity. The mechanisms of injury included road traffic accidents (22 patients, $73 \%$ ); fall from height (four patients, 13\%); and various types of lowvelocity trauma such as, fall from standing height and fall from a moving truck (four patients,13\%). Eight patients $(27 \%)$ presented with isolated fractures and 22 patients $(73 \%)$ presented with various associated injuries (Fig 1). The most common associated injury was limb injuries followed by head injuries. Patients with acetabular fractures tended to present with isolated injuries while patients with pelvic fractures often presented with multiple injuries (Table 2).

There were 12 patients with pelvic fractures and 18 patients with acetabular fractures. In the latter cohort, eight patients presented with fracture-dislocations of the hip. The most common acetabular fractures were elementary posterior wall fractures while the least common were Judet Type C fractures. Amongst the pelvic injuries, the most common subtype was the Tile Type $\mathrm{C} 1$ fracture. The various fracture types in the two cohorts are diagrammatically represented in figure 2.
Patients with pelvic fractures underwent an average of 1.7 surgical procedures. Often, this consisted of an initial temporary external fixation followed by a definitive open reduction and internal fixation. Mean and median time to definitive surgical fixation of pelvic fractures was 7.3 days (range, 0 - 19d) and 7 days respectively. For three patients with isolated open book pelvic fractures, external fixation was chosen as the definitive mode of fixation.

Patients with acetabular fractures underwent an average of 1.8 surgical procedures, often consisting of initial insertion of a Steinmann pin for skeletal traction followed by definitive open reduction and internal fixation. Mean and median time to definitive surgical fixation of acetabular fractures was 11.4 days (range, 3 - 17) and 7.5 days respectively.

There were 17 patients who required a short period of preand post-operative clinical management in a $\mathrm{CCU}$ with a mean and median period of stay of 5.4 days (range, 1 - 22 days) and four days respectively. The median length of hospital stay was 19.5 days (range four to 72 days). Postoperative complications included deep vein thrombosis (1 patient), lateral femoral cutaneous nerve palsy (2 patients) and post-operative infection (5 patients, of which 4 were superficial surgical site infections, which resolved with antibiotic therapy). One patient had an extended clinical stay (72 days) due to post-operative infection complications. He developed a deep surgical site infection with hip osteomyelitis secondary to seeding from a fungal respiratory infection. He required multiple surgical debridements and removal of surgical implants. He eventually underwent a total hip arthroplasty at approximately one year following the initial acetabular trauma.

The time for patients with uncomplicated fracture to heal and to return to full weight bearing ranged from one to 25 weeks (median, 9 weeks; mean, 9.4 weeks). One patient was paraplegic from a concomitant spinal injury and remained non-ambulatory. A total of three patients required total hip arthroplasty for avascular necrosis of the femoral head, severe progressive osteoarthritis, and osteomyelitis of the femoral head (described earlier). One patient was noncompliant for non-weight bearing instructions and eventually developed loosening of the acetabular implants necessitating revision fracture fixation. Excluding the patient who was paraplegic from a concomitant spinal injury, 5 of 29 (17.2\%) patients had poor clinical outcomes despite surgical fracture fixation. D'Aubigne \& Postel Hip scores ranged from 44 to 18 (median, 17). A summary of relevant patient demographics and functional outcomes is summarised in Table 3 . 
In this study, the following determinants correlated with postoperative hip scores: age, number of concomitant injuries and length of stay in a CCU (Table 4). These findings were subjected to statistical analysis using Chisquared tests. Since the patient numbers involved in this study was small, these findings were found to be approaching significance but not at $p<0.05$. Patients with acetabular fractures generally had better functional outcomes (mean hip score 15.0) compared to patients with pelvic fractures (mean hip score 13.5). For patients with acetabular fractures, anatomical reduction was measured and categorized into one of three categories: $<1 \mathrm{~mm}, 1-5 \mathrm{~mm}$ and $>5 \mathrm{~mm}$. Hip scores strongly correlated to the accuracy of reduction, with patients having fracture reduction to within five millimetres having the best scores $(p<0.05)$.

Table $1 \mathrm{~A}$

\begin{tabular}{|lc|}
\hline Radiological Grade & Residual Displacement \\
\hline Good & $<1 \mathrm{~mm}$ \\
Fair & 1 to $5 \mathrm{~mm}$ \\
Poor & $>5 \mathrm{~mm}$ \\
\hline
\end{tabular}

Table 1B

\begin{tabular}{|llll|}
\hline & Pain & Mobility & Ability to walk \\
\hline 0 & Intense \& Permanent & Ankylosis with poor hip position & None \\
1 & Severe with night pain & Nil; slight deformity / pain & Only with crutches \\
2 & Severe when walking & Flexion $<40^{\circ}$ & Only with canes \\
3 & Tolerable with limited activity & Flexion $40-60^{\circ}$ & One cane, $<1 \mathrm{hr}$ \\
4 & Mild walking pain, no rest pain & Flexion $60-80^{\circ}$. , can touch foot & Long time with cane, short time without \\
5 & Mild \& inconstant, Normal activity & $80-90^{\circ} .$, abduction $15^{\circ}$ & No cane, but slight limp \\
6 & No pain & Flexion $>90^{\circ}$. , abduction to $30^{\circ}$ & Normal \\
& & \\
\hline
\end{tabular}

\begin{tabular}{|c|c|c|}
\hline Pain & Ability to walk & \\
\hline & & Very Good $\mathrm{P}+\mathrm{W}=11$ or 12 \\
\hline 6 & 6 & Walk without cane, no pain \& no limp \\
\hline 6 & 5 & Walk without cane, no pain but slight limp \\
\hline 5 & 6 & Walk without cane, no limp but slight pain when starting \\
\hline & & Good $\quad P+W=10$ \\
\hline 5 & 5 & Walk without cane, slight pain \& slight limp \\
\hline 4 & 6 & Walk without cane, with pain but no limp \\
\hline 6 & 4 & Cane used to go outdoors, no pain \\
\hline & & Medium $\quad P+W=9$ \\
\hline 5 & 4 & Slight pain, Cane used outdoors \\
\hline 4 & 5 & Pain after walking a few mins; No cane; Slight limp \\
\hline 6 & 3 & No pain; Cane used all the time \\
\hline & & Fair $\quad P+W=8$ \\
\hline 5 & 3 & Slight pain; Cane used all the time \\
\hline 4 & 4 & Pain after walking; Cane used outdoors \\
\hline & & $\begin{array}{l}\text { Poor } \quad P+W=7 \text { or less } \\
\text { Mobility reduced }\end{array}$ \\
\hline
\end{tabular}

If mobility is reduced to 4 , the result is classed one grade lower

If mobility is reduced to 3 or less, the result is classed two grades lower.

Table 2

\begin{tabular}{|llll|}
\hline Number of concomitant injuries & $\mathbf{0}$ & $\mathbf{1 - 2}$ & $>\mathbf{2}$ \\
\hline Patients with acetabular fractures $(n=18)$ & $7(39 \%)$ & $9(50 \%)$ & $2(11 \%)$ \\
Patients with pelvic fractures $(n=12)$ & $1(8 \%)$ & $8(67 \%)$ & $3(25 \%)$ \\
\hline
\end{tabular}


Table 3

\begin{tabular}{|lcc|}
\hline & Pelvic fractures* & Acetabular fractures \\
\hline Patients ( $\mathrm{n})$ & 11 & 18 \\
Gender (Male/Female) & $5 / 6$ & $15 / 3$ \\
Mean age (years) & 35 & 40 \\
Mean number of surgical procedures & 1.8 & 1.8 \\
Mean time to full weight-bearing status (weeks)** & 9.5 & 15.2 \\
Mean hip scores & 13.5 & 15 \\
\hline
\end{tabular}

* - The patient who was paraplegic secondary to a spinal cord injury has been excluded.

** - Refers to patients who attained full weight-bearing status following the definitive fracture fixation. One pelvic fracture patient and two acetabular fracture patients underwent subsequent total hip replacement.

Table 4A

\begin{tabular}{|lccc|}
\hline Age (years) & $<30$ & 30 to 50 & $>50$ \\
\hline Mean hip scores & 15.4 & 16.1 & 14.1 \\
\hline
\end{tabular}

Table 4C

\begin{tabular}{|lccc|}
\hline Duration of CCU stay & $\mathbf{0}$ days & $\mathbf{1 - 5}$ days & $>$ 5 days \\
\hline $\begin{array}{l}\text { Mean number of } \\
\text { associated injuries }\end{array}$ & 0.8 & 2.3 & 3.7 \\
Mean hip scores & 16.9 & 15.4 & 16 \\
\hline
\end{tabular}

CCU - Critical Care Unit
Table 4B

\begin{tabular}{|lccc|} 
Number of concomitant injuries & $\mathbf{0}$ & $\mathbf{1 - 2}$ & $>3$ \\
\hline Mean hip scores & 17 & 15 & 13
\end{tabular}

Table 4D

\begin{tabular}{|lccc|}
\hline $\begin{array}{l}\text { Residual fracture } \\
\text { displacement }\end{array}$ & $<1 \mathrm{~mm}$ & $1 \mathrm{~mm}$ to $5 \mathrm{~mm}$ & $>5 \mathrm{~mm}$ \\
\hline Mean hip scores & 18 & 16 & 11 \\
\hline
\end{tabular}

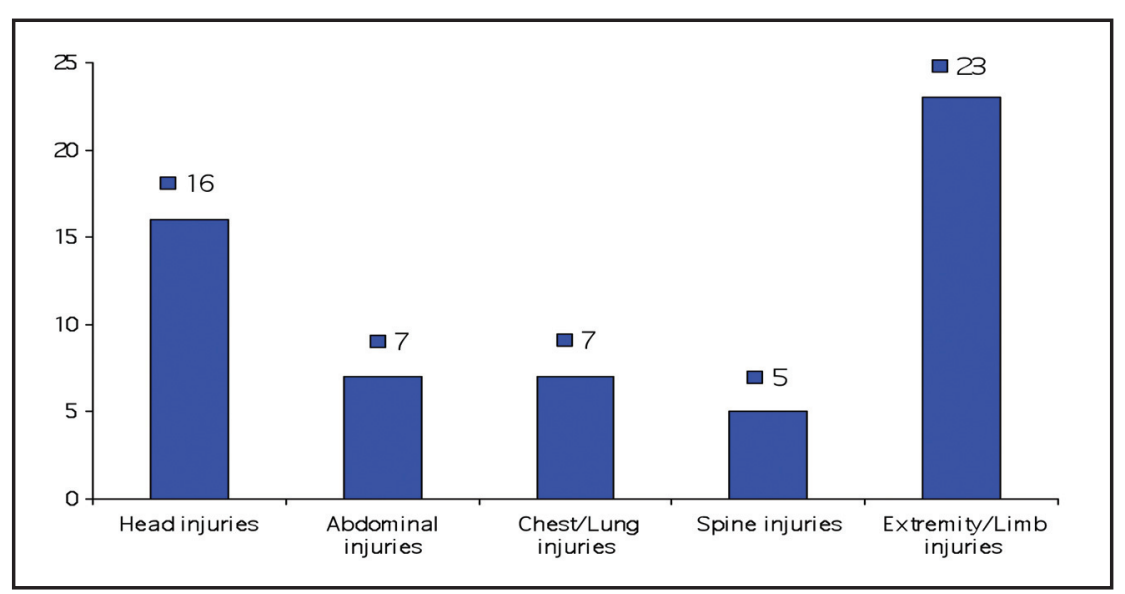

Fig. 1: Pattern of associated injuries.
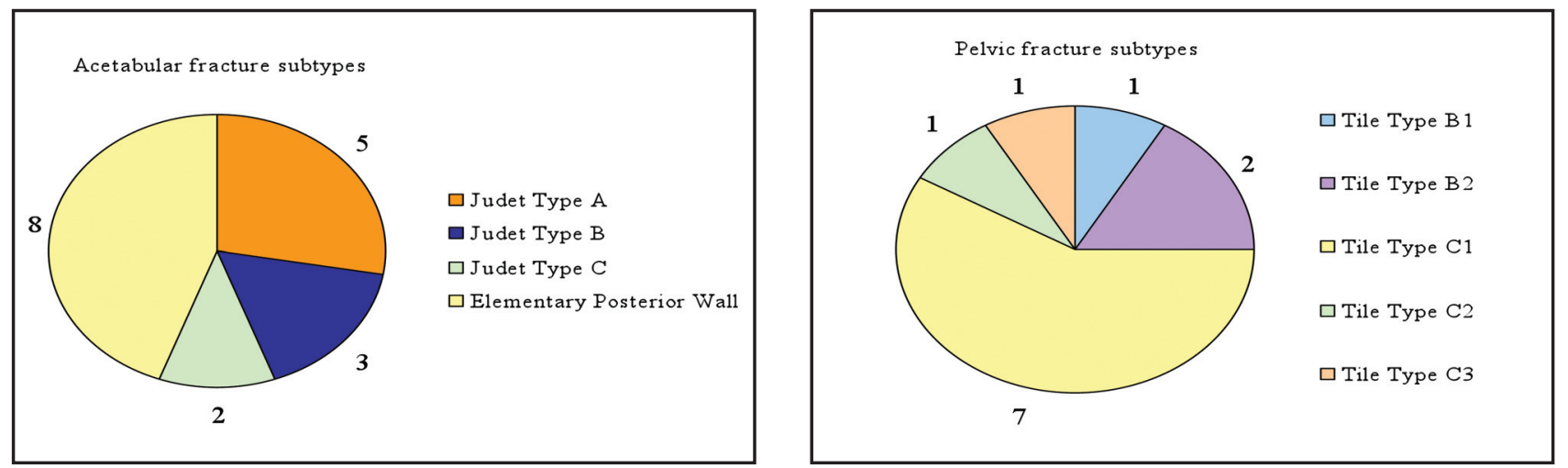

Fig. 2: The various sub-types of acetabular and pelvic fractures 


\section{DISCUSSION}

Early definitive stabilization of pelvic and acetabular fractures is optimal as it facilitates early functional rehabilitation. However, this is often not possible, since patients often have various physiologic insults that require stabilization prior to treating the fracture ${ }^{2,4,5,6,11,12,13,14}$. In acetabular fracture patients, the delay may not always be critical since the clinical outcomes in patients who had early surgery was not significantly different from patients who underwent late (i.e. after 21 days) definitive surgery ${ }^{15}$. In the present study, mean hip scores were significantly better in patients who underwent fixation within 5 days from the time of injury. Early intervention facilitates early limb mobilization and subsequently faster functional recovery. A delay of 5-10 days was reflective of patients with multiple comorbidities that required an extended time period for clinical stabilization. Moreover, surgery during this time period may be associated with greater likelihood of complications due to the catabolic phase following polytrauma ${ }^{16}$. The effect of delay on fracture fixation in patients with pelvic and acetabular fractures was also reviewed by Plaisier et al and their results also demonstrated that early reduction in both groups is associated with a decreased length of stay and complications ${ }^{17}$.

Age was an important determinant of postoperative clinical outcome. This could be attributed to the fact that patients over the age of 50 years tend to have a higher prevalence of co-morbidities including hypertension, diabetes mellitus and hyperlipidaemia, which may have affect rehabilitation potential. Patients younger than 30 years of age had slightly poorer hip scores mainly due to the fact that many were involved in higher energy trauma and sustained concomitant injuries (75\% had concomitant limb injuries, 25\% had serious chest/lung injuries, $43.8 \%$ had concomitant head injuries and $18.8 \%$ had serious spinal injuries).

The duration of stay in CCU also had a bearing on the clinical outcome, and is probably attributable to the fact that the patients requiring a stay in the CCU generally sustained more severe injuries and also had more physiological compromise as a result of a longer duration of relative immobility, which can then affect rehabilitation potential.

Both pelvic and acetabular fractures are secondary to highenergy transfers to strong osseous struts. Due to the involvement of a complex hemi-spherical joint, the rehabilitation and clinical outcomes in acetabular fracture patients would be expected to be poorer than in patients with pelvic fractures. In the current study, hip scores were better in the acetabular fracture group compared to patients with pelvic fractures. This can be attributed to the fact that unlike acetabular fractures, pelvic fractures are typically associated with multiple injuries. Moreover, patients with pelvic fractures may present with significant physiological derangement resulting in poorer long-term functional outcomes as previously reported ${ }^{18,19,20,21}$. However, due to limited sample size, this difference was not statistically significant in the present study.

Acetabular fracture outcomes correlated with age as poorer clinical outcomes were noted in older patients. In the elderly, fixation can be compromised by suboptimal bone stock making fixation tenuous. Moreover, patients may have preexisting degenerative arthritis and the occurrence of trauma increases the risk of clinical deterioration. Moed et al. noted that despite the simplicity of fracture types in the acetabular fractures, results are tempered due to comminution and age ${ }^{12,22}$. Definitive internal fixation of acetabular fractures is often technically demanding and the complexity of the fracture type, age of patients and the development of osteonecrosis can result in poor outcomes in up to $30 \%$ of patients ${ }^{23,24,25}$. Some authors have even recommended the option of acute total hip replacement to address comminuted acetabular fractures in the elderly ${ }^{24,26}$. However, total hip arthroplasty in the setting of compromised soft-tissue and osseous conditions is also fraught with technical difficulties ${ }^{27}$.

Management of acetabular fractures is technically challenging and can be accompanied by numerous complications $^{8}$. Fracture reduction is important to decrease the risk of osteoarthritis ${ }^{29}$. Anatomic reduction with minimal displacement was correlated with good clinical outcomes in the present study and indeed, similar findings have been reported by other authors ${ }^{7,12}$. Although many studies report improved clinical outcomes after better anatomical reduction of acetabular fractures, in the present study, we found that $16.7 \%$ of our patients reported poor clinical outcome despite operative reduction of acetabular fractures. Smaller residual fracture displacement postoperatively was generally associated with better clinical outcome.

\section{CONCLUSION}

Pelvic and acetabular fractures are often a result of high energy trauma and patients with such fractures often have other associated injuries (commonly limb and head injuries) which may adversely affect rehabilitation potential. Fewer concomitant injuries, younger age, and shorter CCU stay resulted in better outcomes. A multicenter study with larger sample size may yield statistical significance for these observations when analyzing these variables. In acetabular fractures however, anatomical reduction was significantly correlated to better functional results.

\section{ACKNOWLEDGEMENT}

The authors thank the following senior surgeons for contributing to this study: Dr Wong Ho Poh, Dr Lai Choon Hin and Dr Sarbjit Singh. 


\section{REFERENCES}

1. O'Sullivan RE, White TO, Keating JF. Major pelvic fractures: identification of patients at high risk. J Bone Joint Surg Br. 2005 Apr; 87(4): 530-3.

2. Routt ML Jr, Simonian PT, Ballmer F. A rational approach to pelvic trauma. Resuscitation and early definitive stabilization. Clin Orthop Relat Res. 1995 Sep; (318): 61-74.

3. Kumar A, Shah NA, Kershaw SA, Clayson AD. Operative management of acetabular fractures. A review of 73 fractures. Injury. 2005 May; 36(5): 605-12.

4. Connor GS, McGwin G Jr, MacLennan PA, Alonso JE, Rue LW 3rd. Early versus delayed fixation of pelvic ring fractures. Am Surg. 2003 Dec; 69(12): 1019-23; discussion 1023-4.

5. Madhu R, Kotnis R, Al-Mousawi A, Barlow N, Deo S, Worlock, P, Willett K. Outcome of surgery for reconstruction of fractures of the acetabulum. The time dependent effect of delay. J Bone Joint Surg Br. 2006 Sep; 88(9): 1197-203.

6. Brueton RN. A review of 40 acetabular fractures: the importance of early surgery. Injury. 1993 Mar; 24(3): 171-4.

7. Matta JM. Fractures of the acetabulum: accuracy of reduction and clinical results in patients managed operatively within three weeks after the injury. J Bone Joint Surg Am. Nov 1996; 78: 1632-45.

8. Matta JM, Tornetta P 3rd. Internal fixation of unstable pelvic ring injuries. Clin Orthop Relat Res. 1996 Aug; (329): 129-40.

9. Tile M. Fracture of the acetabulum. In: Rockwood CA, ed. Rockwood and Green's Fractures in Adults, 4th ed. Philadelphia: Lippincott-Raven Publishers, 1996, 1617-58.

10. Judet R, Judet J, Letournel E. Fracture of the acetabulum: classification and surgical approaches for open reduction: preliminary report. J Bone Joint Surg [Am] 1964, 46: 1615-46.

11. Bircher M, Lewis A, Halder S. Delays in definitive reconstruction of complex pelvic and acetabular fractures. J Bone Joint Surg Br. 2006 Sep; 88(9): 1137-40.

12. Moed BR, Carr SE, Watson JT. Open reduction and internal fixation of posterior wall fractures of the acetabulum. Clin Orthop Relat Res. 2000 Aug; (377):57-67.

13. Oransky M, Sanguinetti C. Surgical treatment of displaced acetabular fractures: results of 50 consecutive cases. $J$ Orthop Trauma. 1993; 7(1): 28-32.

14. Deo SD, Tavares SP, Pandey RK, El-Saled G, Willett KM, Worlock PH. Operative management of acetabular fractures in Oxford. Injury. 2001 Sep; 32(7): 581-6.

15. Matta JM, Tornetta P 3rd. Internal fixation of unstable pelvic ring injuries. Clin Orthop Relat Res. 1996 Aug; (329): 129-40.

16. Hasenboehler E, Williams A, Leinhase I, Morgan SJ, Smith WR, Moore EE, Stahel PJ. Metabolic changes after polytrauma: an imperative for early nutritional support. World J Emerg Surg. 2006; 1:29.

17. Plaisier BR, Meldon SW, Super DM, Malangoni MA. Improved outcome after early fixation of acetabular fractures. Injury. 2000 Mar; 31(2): 81-4.

18. Pohlemann T, Gansslen A, Schellwald O, Culemann U, Tscherne H. Outcome after pelvic ring injuries. Injury. 1996; 27 Suppl 2: B31-8.

19. Van den Bosch EW, Van der Kleyn R, Hogervorst M, Van Vugt, AB. Functional outcome of internal fixation for pelvic ring fractures. J Trauma. 1999 Aug; 47(2): 365-71.

20. Miranda MA, Riemer BL, Butterfield SL, Burke CJ 3rd. Pelvic ring injuries. A long-term functional outcome study. Clin Orthop Relat Res. 1996 Aug; (329):152-9.

21. Korovessis P, Baikousis A, Stamatakis M, Katonis P. Medium- and long-term results of open reduction and internal fixation for unstable pelvic ring fractures. Orthopedics. 2000 Nov; 23(11): 1165-71.

22. Moed BR, WillsonCarr SE, Watson JT. Results of operative treatment of fractures of the posterior wall of the acetabulum. $J$ Bone Joint Surg Am. 2002 May; 84-A(5): 752-8.

23. Fica G, Cordova M, Guzman L, Schweitzer D. Open reduction and internal fixation of acetabular fractures. Int Orthop. 1998; 22(6): 348-51. 
24. Mears DC, Velyvis JH, Chang CP. Displaced acetabular fractures managed operatively: indicators of outcome. Clin Orthop Relat Res. 2003 Feb; (407): 173-86.

25. Liebergall M, Mosheiff R, Low J, Goldvirt M, Matan Y, Segal D. Acetabular fractures. Clinical outcome of surgical treatment. Clin Orthop Relat Res. 1999 Sep; (366): 205-16.

26. Kreder HJ, Rozen N, Borkhoff CM, Laflamme YG, McKee MD, Schemitsch EH, Stephen DJ. Determinants of functional outcome after simple and complex acetabular fractures involving the posterior wall. J Bone Joint Surg Br. 2006 Jun; 88(6): 776-82.

27. Helfet DL, Borrelli J Jr, DiPasquale T, Sanders R. Stabilization of acetabular fractures in elderly patients. J Bone Joint Surg Am. 1992 Jun; 74(5): 753-65.

28. Giannoudis PV, Grotz MR, Papakostidis C, Dinopoulos H. Operative treatment of displaced fractures of the acetabulum. A metaanalysis. J Bone Joint Surg Br. 2005 Jan; 87(1): 2-9.

29. Letournel E. Acetabulum fractures: classification and management. Clin Orthop Relat Res. 1980 Sep; (151): 81-106. 